Relations industrielles

Industrial Relations

\title{
Avis préalable de lock-out
}

\section{Rodrigue Blouin}

Volume 34, numéro 2, 1979

URI : https://id.erudit.org/iderudit/028969ar

DOI : https://doi.org/10.7202/028969ar

Aller au sommaire du numéro

\section{Éditeur(s)}

Département des relations industrielles de l'Université Laval

\section{ISSN}

0034-379X (imprimé)

1703-8138 (numérique)

Découvrir la revue

\section{Citer cet article}

Blouin, R. (1979). Avis préalable de lock-out. Relations industrielles / Industrial Relations, 34(2), 359-369. https://doi.org/10.7202/028969ar

Tous droits réservés @ Département des relations industrielles de l'Université Laval, 1979
Ce document est protégé par la loi sur le droit d'auteur. L’utilisation des services d'Érudit (y compris la reproduction) est assujettie à sa politique d'utilisation que vous pouvez consulter en ligne.

https://apropos.erudit.org/fr/usagers/politique-dutilisation/ 


\section{DROIT DU TRAVAIL}

\section{Avis préalable de lock-out}

\section{Rodrigue Blouin}

Dans un jugement récent ${ }^{1}$ la Cour suprême a unanimement décidé que l'employeur qualifiable de service public peut exercer le lock-out même si le syndicat n'a pas encore donné l'avis écrit de 8 jours prévu à l'article 99 du Code du travail. Ce jugement infirme celui rendu majoritairement par la Cour d'appel ${ }^{2}$ et confirme ceux prononcés par la Cour supérieure (chambre criminelle) ${ }^{3}$ et le Tribunal du travail. ${ }^{4}$

Cette décision apparaît d'une importance pratique considérable même si l'article $99 \mathrm{k}$ adopté récemment ${ }^{5}$ édicte que les employeurs des secteurs public et parapublic doivent donner un avis préalable avant d'exercer le lock-out, avis qui se particularise de celui prévu à l'article 99 .

\section{LE LITIGE}

Les faits à l'origine du litige dont a été saisie la Cour suprême remontent à 1974. En date du 9 novembre l'employeur, qui est un service public au sens de l'article $1 \mathrm{n}$ ) du Code du travail, décréta un lock-out alors que les délais prévus à l'article 46 dudit Code étaient parvenus à terme le 4 novembre. Cependant, il ne donna pas l'avis prévu à l'article 99. Pour fins de mise en contexte, rappelons que ces articles se lisaient à l'époque comme suit:

* BLOUIN, Rodrigue, Professeur de Droit du travail au Département des Relations Industrielles à l'Université Laval et à la Faculté de Droit de l'Université d'Ottawa.

I La Cité de Hull - vs - Le Syndicat des Employés Municipaux de la Cité de Hull Inc., Cour suprême, 5 décembre 1978. Le coram était formé des honorables juges Beetz, Dickson, Estey, Pigeon et Pratte. Les motifs du jugement ont été prononcés par le juge Pratte et y ont souscrit les quatre autre juges.

2 Syndicat des Employés Municipaux de la Cité de Hull Inc. - vs - La Cité de Hull, Cour d'appel, district de Montréal, dossier no 10-000070-761, 14 juin 1977; les honorables juges Bélanger, Lajoie et Owen, ce dernier étant dissident.

3 Syndicat des Employés Municipaux de la Cité de Hull Inc. - vs - Cité de Hull, Cour supérieure (chambre criminelle), district de Hull, no M-75-2166 (Hull - 27-06187), 11 février 1976; l'honorable J. Boucher. Jugement rapporté à (1976) R.D.T. 150.

4 Syndicat des Employés Municipaux de la Cité de Hull Inc. - vs - La Cité de Hull, Tribunal du travail, Montréal, no M-75-2166, 16 mai 1975; monsieur le juge V. Melançon, Décision rapportée à (1975) T.T. 111.

5 Projet de loi 59, 1978, art. 4 (sanctionné le 23 juin 1978). 
$\ll 46$

Si l'intervention du conciliateur a été infructueuse, le droit à la grève ou au lock-out est acquis soixante jours ou, s'il s'agit de la conclusion d'une première convention collective, quatre-vingt-dix jours, après la réception par le ministre de l'avis prévu à l'article 43, à moins que les parties ne soumettent leur différend à un conseil d'arbitrage».

«99

La grève est interdite aux salariés à l'emploi d'un service public à moins que l'association de salariés en cause y ait acquis droit suivant l'article 46 et ait donné par écrit au ministre avis préalable d'au moins huit jours lui indiquant le moment où elle entend y recourir.

Si $(\ldots \ldots \ldots \ldots \ldots)$........

Le lock-out est intervenu du 9 novembre au 3 décembre 1974. Le syndicat n'avait pas alors manifesté au Ministre son intention de recourir à la grève.

Le tribunal du travail fut saisi d'une plainte pénale comportant quatre chefs d'accusation dont le premier, et seul pertinent ici, est libellé en ces termes:

«le ou vers le 9 novembre 1974, dans le district de Hull, a déclaré un lock-out contrairement aux dispositions de l'article 97 du Code du travail commettant ainsi l'infraction prévue à l'article 124 du Code».

Les articles 97 et 124 du Code se lisent comme suit:

\section{$\ll 97$}

Le lock-out est interdit sauf dans le cas où une association de salariés a acquis droit à la grève.»

$\ll 124$

Quiconque déclare ou provoque une grève ou lock-out contrairement aux dispositions du présent code, ou y participe, est passible, s'il s'agit d'un employeur ou d'un officier ou représentant d'une association, d'une amende de cent à mille dollars pour chaque jour ou partie de jour pendant lequel cette grève ou ce lock-out existe et, dans tous les autres cas, d'une amende de dix à cinquante dollars pour chaque tel jour ou partie de jour.»

La compréhension de l'article 97 nécessite de rappeler la teneur de l'article 94:

«94

La grève est interdite tant qu'une association des salariés en cause n'a pas été accréditée et n'y a pas acquis droit suivant l'article 46.»

Selon le plaignant, l'employeur aurait décrété un lock-out illégal. Car, d'une part, le législateur a édicté à l'article 97 que le lock-out n'est permis que dans les cas où un syndicat a acquis droit à la grève. D'autre part, il a stipulé à l'article 99 que le syndicat accrédité regroupant des salariés d'un service public a l'obligation de donner un avis de huit jours indiquant le moment qu'il entend recourir à la grève. Il découlerait de ces textes que le lock- 
out ne peut être légal qu'une fois cet avis donné, et le moment survenu. Le tribunal du travail rejeta la plainte, prononça l'acquittement de l'employeur. Ce jugement fut confirmé par la Cour supérieure (chambre criminelle) devant qui s'était pourvu le syndicat. La Cour d'appel infirma majoritairement ces décisions et trouva l'employeur coupable de l'infraction reprochée. La Cour suprême fut unanimement d'opinion «que le pourvoi doit être accueilli, l'arrêt de la Cour d'appel infirmé et les jugements de la Cour supérieure et du Tribunal du travail rétablis». ${ }^{6}$

\section{LE JUGEMENT DE LA COUR SUPRÊME}

Pour mieux saisir les sens et portée du jugement de la Cour suprême, il apparaît opportun de rappeler d'abord les principaux éléments de celui rendu par la Cour d'appel. Ce dernier jugement s'autorise plus particulièrement d'une décision antérieure, l'affaire Commission Scolaire de Chambly ${ }^{7}$, laquelle n'est cependant pas un cas de plainte pénale mais un cas de requête en évocation. Dans cette affaire de la C.S.R. de Chambly, la Cour d'appel fut d'opinion qu'il n'y avait pas lieu de faire de distinction «entre les conditions préalables requises pour l'acquisition du droit de grève et celles nécessaires à son exercice». ${ }^{8}$ Selon cette Cour, l'article $99 \mathrm{du}$ Code suspend l'acquisition du droit de grève, et non pas simplement son exercice, jusqu'à ce que l'avis de huit jours soit donné. Et de rajouter la Cour d'appel, "l'Association n'avait pas acquis le droit à la grève, qu'à tout événement elle ne pouvait l'exercer si elle l'avait, et qu'en conséquence l'employeur n'avait pas non plus acquis le droit au «lock-out»ou tout au moins ne pouvait l'exercer». ${ }^{9}$ Le motif essentiel qui semble avoir fondé cette prise de position est exprimé en ces termes par le juge Lajoie:

«Il est déraisonnable de penser que le législateur qui a lié ensemble dans le Code du Travail ces droits de l'employeur et de l'employé, ait voulu que l'employeur puisse exercer le droit au «lock-out» avant que l'association de salariés ne puisse exercer son droit de la grève. L'intention de l'article 97, lu en regard de l'article 46, est clairement au contraire». ${ }^{10}$

La cour a conclu que puisque l'employeur n'avait pas acquis le droit au «lock-out», une modification aux conditions de travail pouvait donner lieu à un grief et alors l'"arbitre décidera s'il y a matière à grief et si tel est le cas, s'il doit être admis ou non". ${ }^{11}$

6 Note 1 , à p. 5 du jugement.

7 Commission Scolaire Régionale de Chambly et Honorable E. Marier et Association des Enseignants de Chambly et Honorable M. Bellemare, R. Mireault, C. Nappert et F. Fortin, Cour d'appel, district de Montréal, no 14340, 22 août 1974, les honorables Brossard, Lajoie et Rinfret. Les motifs ont été énoncés par le juge Lajoie auquel ont concourru les deux autres juges. Le jugement est rapporté à: (1976) R.D.T. 129.

8 Note 7, à p. 142.

9 Ibidem, à p. 143.

10 Ibidem, à p. 143.

11 Ibidem, à p. 149. 
Dans la cause Cité de Hull, le juge Bélanger de la Cour d'appel signala qu'il avait lu les notes du juge Lajoie dans l'affaire C.S.R. de Chambly et mentionna ce qui suit:

«Il est bon de noter ici qu'une demande d'autorisation d'appel à la Cour suprême du Canada du jugement rendu dans l'affaire de la Commission Scolaire Régionale de Chambly, fut refusée. Sa portée comme précédent garde donc toute sa valeur dans les limites de notre droit sur le «stare decisis». Face à l'interprétation de notre Cour, le législateur n'a pas jugé opportun de modifier sa loi. Mais il me suffit de dire dans l'espèce que je partage l'opinion exprimée par mon collègue Monsieur le Juge Lajoie dans le dit jugement». ${ }^{12}$

Le juge Bélanger fit cependant quelques commentaires additionnels et dont l'un est d'une importance capital. Selon ce juge, c'est carrément l'interdiction du lock-out qui persiste tant que la grève est interdite aux salariés. Ainsi, le lock-out serait interdit tant que le syndicat n'a pas donné l'avis préalable de grève prévu à l'article 99 . Il n'est donc nullement question que l'employeur puisse donner au ministre un avis préalable d'exercice de son droit de lock-out. Le juge s'est exprimé comme suit:

«Il convient ici de préciser que dans l'arrêt antérieur de cette Cour, Commission Scolaire Régional de Chambly, il n'est aucunement question que l'employeur doive donner un tel avis pour que l'interdiction de l'article 97 tombe. La portée réelle dudit arrêt, de même que celle de la présente opinion, c'est que l'interdiction du lock-out persiste tant que la grève est interdite aux salariés aux termes de l'article 99 lorsqu'il s'agit d'un service public; c'est à un avis par l'association de salariés que cet article réfère». ${ }^{13}$

Le juge Lajoie fut pour sa part d'opinion que l'on ne l'avait pas convaincu que ses propos dans l'affaire C.S.R. de Chambly devaient être modifiés et se déclara d'accord avec le juge Bélanger et pour les mêmes motifs. Le juge Owen enregistra sa dissidence aux avis exprimés par les juges Lajoie et Bélanger. Le motif essentiel du juge Owen est le suivant:

"In the present case we are dealing with a penal action and the Respondent should not be convicted unless it has infringed the text of the law. There is no text in the Labour Code which states that an employer in a public service cannot declare a lock-out unless the association of his employees has given an light days notice ...". ${ }^{14}$

Et immédiatement le juge poursuit en ces termes:

"Such a text would place in the hands of the association of employees the power to determine when and whether a lock-out could be declared by the employer. If any doubt raised by Section 99 , which deals with

12 Note 2, à p. 5 des notes du juge Bélanger.

13 Ibidem, aux pp. 6 et 7.

14 Ibidem, aux pp. 5 et 6 des notes du juge Owen. 
strikes by employees of a public service and not with lock-outs, such doubt should be resolved in favour of the person who would be liable to a penalty under the statute". 15

Pour la Cour suprême, la décision majoritaire de la Cour d'appel est erronée et ce, pour deux motifs principaux. D'abord parce que la Cour d'appel aurait interprété l'article 97 du Code du travail comme si l'interdiction valait pour tous les cas où la grève est interdite plutôt que pour les seuls cas où un syndicat n'a pas encore acquis droit à la grève. Selon la Cour suprême, l'article 97 «impose l'interdiction sauf dans le cas où une association de salariés a acquis droit à la grève et non pas dans le cas où la grève est interdite». ${ }^{16}$ Comme second motif, la Cour suprême est d'avis que la décision majoritaire de la Cour d'appel ignore la distinction faite par le législateur «entre l'acquisition du droit de grève et les modalités préalables à son exercice». ${ }^{17}$ Ainsi, pour la Cour suprême, s'il est exact que le législateur a encadré de façon particulière l'exercice du droit de grève dans les services publics, il apparaît de même tout aussi exact que le législateur n'a pas assujetti l'exercice du droit de lock-out à de semblables particularités. Le législateur n'a pas imposé de distinction entre l'acquisition du droit de lockout et son exercice. Ce droit est acquis en même temps que le droit à la grève et peut être exercé dès cet instant:

«(...) le lock-out cesse d'être interdit en vertu de l'art. 97 lorsque l'association de salariés a acquis droit à la grève suivant l'art. 46, même si cette association n'a pas encore donné l'avis écrit de huit jours prévu à la dernière partie du premier alinéa de l'art. 99».18

Par son jugement, la Cour suprême rétablit ceux de la Cour supérieure et du Tribunal du travail.

Au sujet du jugement rendu par la Cour supérieure dans cette affaire de la Ville de Hull, il peut être d'intérêt de noter que le juge Boucher avait cerné la question à débattre en des termes autres que ceux utilisés par la Cour suprême et la Cour d'appel:

«L'appelant est-il justifié de prétendre que la condition sine qua non de l'avis préalable de huit jours dans le cas des salariés à l'emploi d'un service public s'applique à l'employeur qui se prévaut de son droit acquis au lock-out?» ${ }^{19}$

S'autorisant d'une règle d'interprétation en matière pénale et voulant que l'on ne peut rajouter des mots à un texte ou en retrancher, le juge estima que l'article 99 a pour but exclusif de réglementer l'exercice du droit à la grève et non pas de réglementer le lock-out une fois que le droit à celui-ci est acquis 
par l'employeur dans les conditions prévues par l'article 97. Pour le juge Boucher,

«Faire droit à la prétention de l'appelant équivaudrait à ajouter un paragraphe au texte de l'article 99, à l'effet que dans le cas de droit acquis au lock-out, l'employeur est tenu de donner au ministre un avis préalable d'au moins 8 jours, lui indiquant le moment où l'employeur entend y recourir ${ }^{20}$

Quant à la décision du juge Melançon du Tribunal du travail, on peut y lire qu' «Il est évident que l'employeur d'un service public n'a pas à donner l'avis de huit (8) jours au Ministre de son intention de décréter un lock-out. Dès qu'il acquiert ce droit, il peut y recourir sans autre formalité. Aucun texte ne le lui impose». ${ }^{21}$ Après une étude très poussée de la jurisprudence et de la doctrine pertinente, le juge Melançon conclut que l'avis préalable de grève est une condition imposée à l'exercice du droit de grève et non pas à son acquisition:

"Cette acquisition du droit de grève se fait au temps indiqué au texte même de l'article 99 du Code du travail, c'est-à-dire à l'expiration du délai mentionné à l'article 46 du Code du travail. Comme l'article $97 \mathrm{du}$ Code du travail indique d'autre part que le droit au lock-out s'acquiert au même moment, mais n'est pas soumis à l'une ou l'autre des modalités d'exercice il faut conclure qu'un lock-out décrété à l'expiration du délai de l'article 46 du Code du travail est parfaitement légal».22

En cela, le juge Melançon rejoignait une opinion exprimée par le juge Marcotte dans une affaire antérieure et de même nature. ${ }^{23} \mathrm{Ce}$ dernier avait affirmé que l'article 99 n'a pas pour conséquence de prolonger le délai utile pour obtenir le droit à la grève ou au lock-out. Selon le juge Marcotte, tout ce que le législateur a édicté à l'article 99 est la suspension du droit de grève tant et aussi longtemps que l'avis prévu n'a pas été donné; le législateur n'a imposé nulle part au Code du travail semblable condition en matière de lock-out. ${ }^{24}$

En définitive, la Cour suprême a confirmé l'approche voulant que le législateur n'a pas fait de distinction entre l'acquisition du droit du lock-out et l'exercice de ce droit alors qu'une telle distinction existe pourtant dans le cas de la grève. De façon indirecte, le jugement de cette Cour implique que l'employeur d'un service public n'a pas à donner un avis préalable de lockout. De façon directe, le jugement est à l'effet que le lock-out cesse d'être interdit, en vertu de l'article 97 du Code, lorsque le syndicat a acquis droit à la grève conformément à l'article 46 même si ce syndicat n'a donné aucun avis de son intention de faire la grève selon l'article 99 .

\footnotetext{
$20 \quad$ Ibidem, aux pp. 155 et 166.

Note 4, à p. 122.

Ibidem, aux pp. 124 et 125 .

J. Cadieux vs. Cité de Ste-Thérèse, (1971) T.T. 220.

Ibidem, à p. 222.
} 


\section{COMMENTAIRES}

Ce jugement de la Cour suprême est d'une importance capitale, principalement pour les(le) services publics au sens du Code du travail qui n'entrent pas dans la catégorie dite secteurs public et parapublic. En effet, un nouvel article, $99 \mathrm{k}$, fait obligation aux employeurs des secteurs public et parapublic de donner un avis préalable de lock-out:

«99k

Sous réserve du quatrième alinéa de l'article 991, une partie peut déclarer une grève ou un lock-out à la date d'expiration de la convention collective ou de ce qui en tient lieu pourvu qu'un avis préalable d'au moins deux jours ait été donné par écrit au ministre et à l'autre partie leur indiquant le moment où elle entend y recourir.

Cet avis de grève ou de lock-out ne peut être renouvelé qu'après le jour indiqué dans l'avis précédent comme moment ou elle entendait recourir à la grève ou au lock-out».

Cet article 99k ne s'applique pas à l'égard de tous les services publics au sens de l'article $1 \mathrm{n})$. Selon l'article $99 \mathrm{a}$, "(...) les dispositions du présent code s'appliquent aux relations du travail dans les secteurs public et parapublic, sauf dans la mesure où elles sont inconciliables avec celles du présent chapitre». (i.e. chap. V.A., art. 99a à 991 - Dispositions particulières applicables aux secteurs public et parapublic). La notion de secteurs publics et parapublic est cernée à l'article 99b):

«Dans le présent chapitre, on entend par «secteurs public et parapublic»: le gouvernement, ses ministères et les organismes du gouvernement dont le personnel est nommé ou rémunéré suivant la Loi de la fonction publique, ainsi que les collèges, les commissions scolaires et les établissements visés par la Loi sur l'organisation des parties patronale et syndicale aux fins des négociations collectives dans les secteurs de l'éducation des affaires sociales et des organismes gouvernementaux».

En comparant ce texte avec celui de l'article $1 \mathrm{n}$ ), on peut constater qu'il y a des services publics au sens du Code du travail qui n'entrent pas dans la catégorie dite des secteurs public et parapublic:

«1n)

-«services publics» - les catégories suivantes d'employeurs:

1e: les corporations municipales et scolaires et le Conseil scolaire de l'Ile de Montréal;

2e: les hôpitaux, les sanatoriums et les institutions pour malades mentaux;

3e: les hospices, les crèches et les orphelinats;

4e: les universités, les collèges et les couvents;

5e: les entreprises de téléphone, de télégraphe, de transport par bateaux, tramways, autobus ou chemins de fer; 
6e: les entreprises de production, transport, distribution ou vente de gaz, d'eau ou d'électricité et les services de transport par véhicules de livraison munis d'une autorisation de la Régie des transports;

7e: les entreprises d'enlèvement d'ordures ménagères;

$8 \mathrm{e}$ : les services du gouvernement de la province et les autres agents de samajesté du chef de la province, à l'exception de la Régie des alcools du Québec».

En raison de cette non-concordance entre les notions de services publics et secteurs publics et parapublic, il appert que la décision de la Cour suprême conserve un intérêt pratique certain pour les employeurs de services publics qui ne font pas partie des secteurs public et parapublic. L'intérêt de ce jugement réside cependant avant tout en ce qu'il atteste que l'exercice d'un droit reconnu à une personne ne peut pas dépendre, sauf texte exprès à l'effet contraire, de la discrétion d'une autre personne. L'interprétation qu'avait donnée la Cour d'appel impliquait que l'exercice du droit au lockout dépendait de ce que ferait le syndicat. Ce point avait été relevé par le juge Melançon du Tribunal du travail ${ }^{25}$ et par le juge Owen, dissident en Cour d'appel ${ }^{26}$, lesquels avaient estimé qu'il s'agirait là d'une situation contraire aux principes généraux du droit. Seul un texte explicite permettrait en effet d'en arriver à la conclusion retenue par la Cour d'appel. Selon la Cour suprême, les textes du Code du travail n'autorisent pas une interprétation en ce sens. Ce jugement nous semble bien fondé.

En effet, selon l'article 97, «Le lock-out est interdit sauf dans le cas où une association de salariés a acquis droit à la grève». On remarquera que ce texte débute par l'expression "Le lock-out» et non pas l'expression "Le droit de lock-out». Par ailleurs, dans la finale on parle bien de «droit de grève». Or le lock-out est précisé à l'article li) comme étant «le refus par un employeur de fournir du travail à un groupe de salariés à son emploi en vue de les contraindre à accepter certaines conditions de travail ou de contraindre pareillement des salariés d'un autre employeur». La définition met en évidence le «fait matériel». Ainsi, l'article 97 ne peut que signifier que le fait matériel du lock-out, son exercice, est interdit ... sauf dans le cas où un syndicat a acquis le droit à la grève. Or les conditions d'acquisition du droit de grève sont précisées essentiellement à l'article 46 et non pas à l'article 94 . L'article 94 stipule que «la grève est interdite tant qu'une association des salariés en cause n'a pas été accréditée et n'y a pas acquis droit suivant l'article 46». Ce texte est le pendant de l'article 97. On dit bien au début du texte «la grève est interdite» et non pas le «droit de grève est interdit». La grève est cernée à l'article $1 \mathrm{~h}$ ) en ces termes: «La cessation concertée de travail par un groupe de salariés». Ainsi l'article 94 ne peut que vouloir dire que le fait matériel de la grève, son exercice est interdit ... tant que le syndicat n'est pas accrédité et «n'y a pas acquis droit suivant l'article 46». On notera que même la finale de ce texte 94 renvoie à l'article 46 pour les fins d'acquisition du droit. Enfin, le texte 46 est des plus explicite: "Le droit à la grève ou au

25 Note 4 , à p. 123.

26 Note 2, à p. 6 des notes du juge Owen. 
lock-out est acquis (...)». Ce sont là les règles générales qui concernent l'acquisition et l'exercice des droits de grève et de lock-out. Par ailleurs, l'article 99 comporte une règle particulière lorsque des services publics au sens de l'article 1n) sont en cause. Cet article 99 débute par l'expression «La grève est interdite (...). Il faut ici aussi comprendre que le fait matériel de la grève, son exercice, est interdit. Le texte rajoute par ailleurs que pour que l'interdiction soit levée, il faut que le droit de grève ait été acquis selon l'article 46 et qu'un avis préalable ait été donné. Il faut donc donner un avis pour exercer la grève; il ne suffit pas d'en avoir acquis le droit. Ce texte 99 en est un dérogatoire aux règles générales énoncées à l'article 94 . Nulle part dans cet article 99, il n'est question du lock-out. Nulle part dans le Code on ne retrouve pour le texte 99 (grève) un pendant visant le lock-out comme on retrouve à l'article 97 (lock-out) le pendant de l'article 94 (grève). De ces observations, il résulte donc que la Cour suprême a redonné plein effet aux principes généraux du droit et aux textes 46, 94, 97 et 99 en cassant la décision pour le moins surprenante de la Cour d'appel.

L'examen des articles 46, 94, 97 et 99 permet de comprendre qu'en vertu des règles générales (art. 46, 94 et 97) il y a coincidence entre l'acquisition du droit à la grève et du droit de lock-out et leur exercice. En vertu de la règle particulière (art. 99) applicable au seul syndicat regroupant des salariés d'un service public, la coincidence entre l'exercice du droit de grève et de lock-out n'existe pas. La doctrine a d'ailleurs signalé ces faits. ${ }^{27}$ Cette solution, même si elle apparaît conforme aux textes, soulève sur le plan politique un problème crucial. Il est en effet de l'essence même de notre système de rapports collectifs que les parties soient placées sur un pied d'égalité non seulement pour les fins de la négociation mais aussi pour l'exercice des moyens de pression économique. Ces données sont fondamentales et leur non-respect dénature carrément le système. Or il appert que dans le cas des services publics, le législateur n'a pas maintenu cet équilibre fondamental qui reste présent dans le cas du secteur privé. Il aurait apparu souhaitable que le législateur rétablisse cet équilibre entre l'exercice du lock-out et de la grève dans le cadre des services publics, en assujettissant l'un et l'autre aux mêmes règles. On pourrait par ailleurs croire que cet équilibre a été rétabli dans le cas des services publics qui appartiennent aux secteurs public et parapublic puisque l'article $99 \mathrm{k}$ prévoit un avis préalable de lock-out. Il n'en est rien en termes d'approche globale et systématique. En effet, cet article $99 \mathrm{k}$ édicte «sous réserve du quatrième alinéa de l'article 991 1》. Or cet alinéa se lit comme suit: "Le droit au lock-out n'est pas acquis si les ententes ou les listes couvrant l'établissement sont respectées (...)»". C'est donc dire que dans le secteur des affaires sociales et dans ce seul secteur, le droit au lock-out n'existe pas en situation normale.

En somme, si l'on tente un essai de synthèse sur les règles gouvernant l'exercice du lock-out dans les services publics sous l'angle de l'avis

27 MORIN, F., Les rapports collectifs du travail au Québec, 1978, Thémis, à p. 174. CUTLER P., Le Code du travail du Québec, 1976, Editions Toundra, aux pp. 276, 418 et 422. GAGNON, LEBEL et VERGE, Droit du Travail en vigueur au Québec, 1971, P.U.L., aux pp. 203,204 et 211 . 
préalable de lock-out, on constate trois coordonnées: $1^{\circ}$ : dans le cas des employeurs des services publics qui ne font pas partie des secteurs public et parapublic, l'article 99 du Code du travail n'est pas applicable; ce texte ne vise que les syndicats. Ainsi, aucun avis préalable de lock-out n'a à être donné. L'exercice du lock-out ne coincide pas nécessairement avec celui de la grève, bien qu'il y ait coincidence sur le moment d'acquisition de l'un et l'autre droit. C'est là le sens de la décision de la Cour suprême. $2^{\circ}$ : dans le cas des employeurs des services publics qui appartiennent aux secteurs public et parapublic mais qui ne font pas partie du réseau des affaires sociales (établissements), le droit au lock-out existe mais un avis préalable de lock-out doit être donné. Il y a coincidence entre acquisition des droits de grève et de lock-out et leur exercice. C'est là le sens des articles 99a, 99b, et $99 \mathrm{k}$ qui excluent l'application de l'article 99 sous la question de l'avis. 3 : dans le cas des employeurs du réseau des affaires sociales, le droit de lockout n'existe pas en situation normale. C'est là le sens des articles $99 \mathrm{k}$ et 991 , al. 4.

Ces règles régissant l'exercice du lock-out attestent d'une certaine complexité. Si on les compare à celles encadrant l'exercice de la grève, elles peuvent être l'occasion de débats épineux. En effet, la coincidence entre l'acquisition des droits de grève et de lock-out et leur exercice est une des pierres angulaires de notre système de rapports collectifs du travail. Or cette coincidence normale et nécessaire tend à s'effriter. Ainsi, les conditions générales d'exercice du droit de grève démontrent que le syndicat doit être autorisé par les salariés à faire la grève et en faire part au ministre (article 19b, al. 1 et 3). S'il déclare la grève, il doit informer le ministre et lui donner les informations requises (article 46a). On ne retrouve comme pendant de cette sorte en matière de lock-out que l'obligation faite à l'employeur d'informer le ministre (article 46a). Ces règles générales sont par ailleurs applicables à tous les services publics. De plus, en vertu des règles particulières, les syndicats des services publics qui n'appartiennent pas aux secteurs public et parapublic voient leur droit de grève suspendu à défaut d'avis préalable de grève ou lorsque la grève risque de mettre en danger la santé ou la sécurité publique (article 99). Il n'y a aucune disposition de cette nature en ce qui concerne le lock-out. Quant aux syndicats des secteurs public et parapublic, ils sont soumis à certaines obligations qui, cette fois, trouvent un pendant du côté du lock-out (article 99k et 991). Par contre, le lock-out est interdit aux employeurs du réseau des affaires sociales lorsque les services essentiels sont maintenus (article 991). L'ensemble de ces règles forment un tout susceptible de laisser comprendre que la coincidence entre les règles régissant l'exercice éventuel de la grève et du lock-out n'est plus qu'apparent.

En un tel contexte, il s'impose que le législateur rétablisse l'équilibre entre les parties. Pour notre part, nous estimons que les contraintes devraient être limitées au maximum. A tout le moins, si des exigences administratives expliquent la présence de certaines dispositions (statistiques, etc.), leur non-respect ne devrait pas entraîner illégalité de la grève mais essentiellement une sanction pénale (amende). Cette solution existe dans le cas du non-respect des prescriptions portant sur le vote d'autorisation de la grève (articles 19b et 19d). Cette même approche devrait être retenue pour 
les autres textes (par exemple, pour le cas de l'article 46a). De plus, nous estimons que le fait de priver les employeurs du réseau des affaires sociales du droit de lock-out en situation normale est un véritable «cheval de Troie» en milieu des relations de travail. Aujourd'hui on enlève le droit de lockout. Demain on enlèvera le droit de grève sous prétexte de rétablir l'équilibre. Dans son récent ouvrage, le professeur Morin posait la problématique qui peut expliquer la disparition du droit du lock-out dans les services publics en général:

\begin{abstract}
«(...) soutenir que l'employeur a droit de faire un lock-out aux conditions de l'art. 99 C.T. semble le placer à contre-courant de la conduite qui lui est imposée auparavant selon cette même disposition. En effet, l'économie même de cet art. 99 impose plusieurs moyens afin d'éviter la grève. A cette fin, l'employeur est implicitement forcé de soutenir préalablement une thèse qui apparaît par la suite irréconciliable à toute décision de faire un lock-out, c'est-à-dire de discontinuer un service public. Dans les circonstances, il s'agit là d'une question qui devrait être clarifiée par le législateur, à savoir si dans le cas d'un service public, un employeur peut effectivement faire un lock-out?».28
\end{abstract}

Pour notre part, nous croyons qu'il serait préférable que le législateur encadre l'exercice du droit de lock-out dans les différents services publics de façon uniforme et selon les mêmes coordonnées qui régissent la grève. Car s'il est dans une certaine mesure exact que dans le cadre des articles 99 et 991 «l'employeur est implicitement forcé de soutenir préalablement une thèse qui apparaît par la suite irréconciliable à toute décision de faire un lock$o u t »$, il n'en reste pas moins que c'est le Lieutenant-gouverneur et lui seul qui a l'initiative de déclencher le mécanisme de suspension de la grève. Et ce qui motive le Lieutenant-gouverneur est le fait que de discontinuer un service public (arrêt de travail) met en cause la santé et la sécurité publique. Si ce type de raisonnement est utilisé pour promouvoir l'interdiction du droit de lock-out, nous le répétons, demain ce même raisonnement sera utilisé pour revendiquer l'interdiction du droit de grève. En un mot, si le législateur estime que les relations du travail dans les services publics peuvent s'accomoder de l'économie qui sous-tend le Code du travail, il s'impose que le législateur rétablisse l'équilibre entre les parties dans l'exercice des moyens de pression économique. Si tel n'est pas le cas, que l'on ait l'honnêteté de créer de toute pièce un système particulier et original qui tiendra compte de la nature des services publics. 